\title{
PHASE LOCK-IN LASER ACTIVE PYROMETRY FOR SURFACE LAYER CHARACTERISATION OF TOKAMAKS WALLS
}

\author{
by D. Melyukov*, C. Sortais*, A. Semerok* , P.Y. Thro*, X. Courtois**, D. Farcage*
}

\author{
${ }^{*}$ CEA Saclay. DEN/DANS/DPC/SCP/LILM \\ ${ }^{*}$ CEA Cadarache, DSM/IRFM/SIPP
}

\begin{abstract}
A lock-in method of surface layer characterization of tokamak walls is presented for determining the thermo-physical properties of a layer (like adhesion, thickness and others) deposited on a substrate, by comparing the experimental results of the phase shift between an excitation signal from a modulated laser and the thermal response of the layer, with the predicted phase shift obtained by a theoretical 3D model. A fast method of phase shift calculations for 3D analytical model was developed. The model validation is done on the stainless steel sheets of different thicknesses and the main experiments were performed on an ITER-like sample.
\end{abstract}

\section{Introduction}

In modern fusion reactors, the erosion of the plasma facing surfaces results in dust deposition on the tokamak "cold" surfaces, in form of layers which could trap tritium. Metallic surface coatings are also used on some components. To provide efficient operation of tokamaks, it is essential to characterize such layers. In-situ fast surface characterization without the reactor component disassembly is required. The lock-in pyrometry appears as a very suitable method: together with a laser (1 Hz - $1 \mathrm{kHz}$ repetition rate frequency), applied for surface heating it can be used to characterize the micrometric layer properties, for example its adhesion to the substrate, its thickness and others.

The lock-in method is based on the propagation of thermal waves in the material and its interaction with discontinuities or non homogeneities. The depth penetration of thermal waves depends on their frequency. This makes it possible to study thermo-physical properties of materials. Any thermal wave has amplitude and phase. In case of contactless temperature measurements by pyrometry method, the amplitude strictly depends on the surface emissivity, heat flux intensity and other optical parameters that are unknown. But the phase of thermal wave is independent of these parameters. This advantage is often used in the thermal characterization of the materials.

Local laser heating of a layer causes a 3D thermal conduction regime and thus a 1D model becomes not valid. On the other hand the 3D theoretical models calculation takes very long time especially in the case of numerical solution. A fast method of phase shift calculations for the analytic theoretical 3D model of the laser heating of the layer was developed [1,2]. Comparison of both theoretical and experimental results makes it possible to determine the main layer parameters. The lock-in pyrometry system was developed and tested on a tile samples $(140 \mu \mathrm{m} \mathrm{W}$-layer on CFC substrate and graphite tile with deposited layer of 10-40 $\mu \mathrm{m}$ thickness of different adhesion).
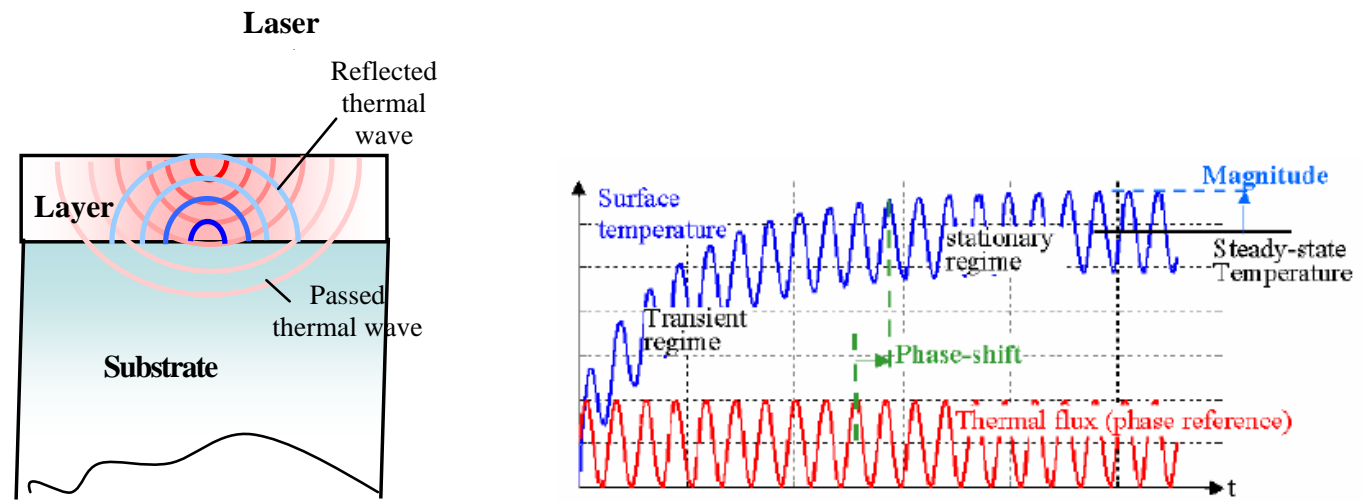

Figure 1. Principe of the lock-in method and thermal wave description. 


\section{Theoretical model}

In the case of a complex sample with a layer of thickness $\Delta$ deposited on the semi-infinite substrate with a contact thermal resistance $\mathrm{R}$ at the substrate/layer interface (which characterizes the layer adhesion quality), the theoretical phase shift between the surface temperature and the heat source at the laser beam center at a repetition rate $f$ can be found from the expression [2]:

$$
\begin{aligned}
\varphi(f) & =\arg \int_{0}^{\infty} \frac{0.5 \xi \cdot e^{-0.25 \xi^{2}}}{\left(\frac{\xi}{r_{0}}\right)^{2}-i 2 \pi f \frac{C_{C} \rho_{C}}{k_{C}}-\alpha^{2}}\left[\frac{2 \alpha \cdot e^{-\kappa_{1} \Delta}}{\kappa_{1} D}\left\{\left(e^{-\alpha \Delta}-e^{-\kappa_{1} \Delta}\right)\left(1+R k_{S} \kappa_{2}\right)+\left(e^{-\kappa_{1} \Delta}-\frac{\kappa_{1}}{\alpha} e^{-\alpha \Delta}\right) \frac{k_{S} \kappa_{2}}{k_{C} \kappa_{1}}\right\}+\left(1-\frac{\alpha}{\kappa_{1}}\right)\right] d \xi \\
D & =\left(1+e^{-2 \kappa_{1} \Delta}\right) \frac{k_{S} \kappa_{2}}{k_{C} \kappa_{1}}+\left(1-e^{-2 \kappa_{1} \Delta}\right)\left(1+R k_{S} \kappa_{2}\right), \kappa_{1}=\sqrt{\left(\frac{\xi}{r_{0}}\right)^{2}-i 2 \pi f \frac{C_{C} \rho_{C}}{k_{C}}}, \kappa_{2}=\sqrt{\left(\frac{\xi}{r_{0}}\right)^{2}-i 2 \pi f \frac{C_{S} \rho_{S}}{k_{S}}} \cdot(1)
\end{aligned}
$$

Hypothesis: isotropic materials, convection neglected at sample surface, linearity.

$\rho_{C^{\prime}} C_{C^{\prime}} k_{C}$ and $\rho_{s}, C_{s}, k_{s}$ are density, mass specific heat, and thermal conductivity of the layer and the substrate, respectively; $\alpha$ is the laser absorption coefficient of the layer; $r_{0}$ is Gaussian laser spot radius at the level 1/e.

\section{Experimental setup}

The schematic view of the experimental setup is shown in Fig. 2. The heat flux is generated at the surface of the deposited layer using a fiber laser of $1080 \mathrm{~nm}$ wavelength and a modulated power driven from frequency function generator. The laser beam has a Gaussian profile and $5 \pm 1 \mathrm{~mm}$ diameter at 1/e.

The thermal response is measured using a pyrometer KLEIBER KGA 740-LO. Its measurement wavelength range is $1.6-2.2 \mathrm{~mm}$ and the diameter of the spot of measurements is $0.5 \mathrm{~mm}$. As The pyrometer is slightly sensitive at the laser wavelength, a high pass optical filter is used to eliminate laser parasitic thermal signal. Time constant of the pyrometer is $10 \mu \mathrm{s}$ and it makes possible to measure the thermal signals of the frequencies below $10 \mathrm{kHz}$. On the other hand the laser power becomes unstable for the pulse frequencies below $10 \mathrm{~Hz}$. So the optimal frequency band is $10-10000 \mathrm{~Hz}$. A lock-in amplifier is used to measure the signal's phase shift between the heating source measured by a photodiode after reflection on a mirror and the thermal response.

In order to increase the signal-to-noise ratio of the pyrometer the samples are heated. For this purpose a sample holder with Joule effect is used.

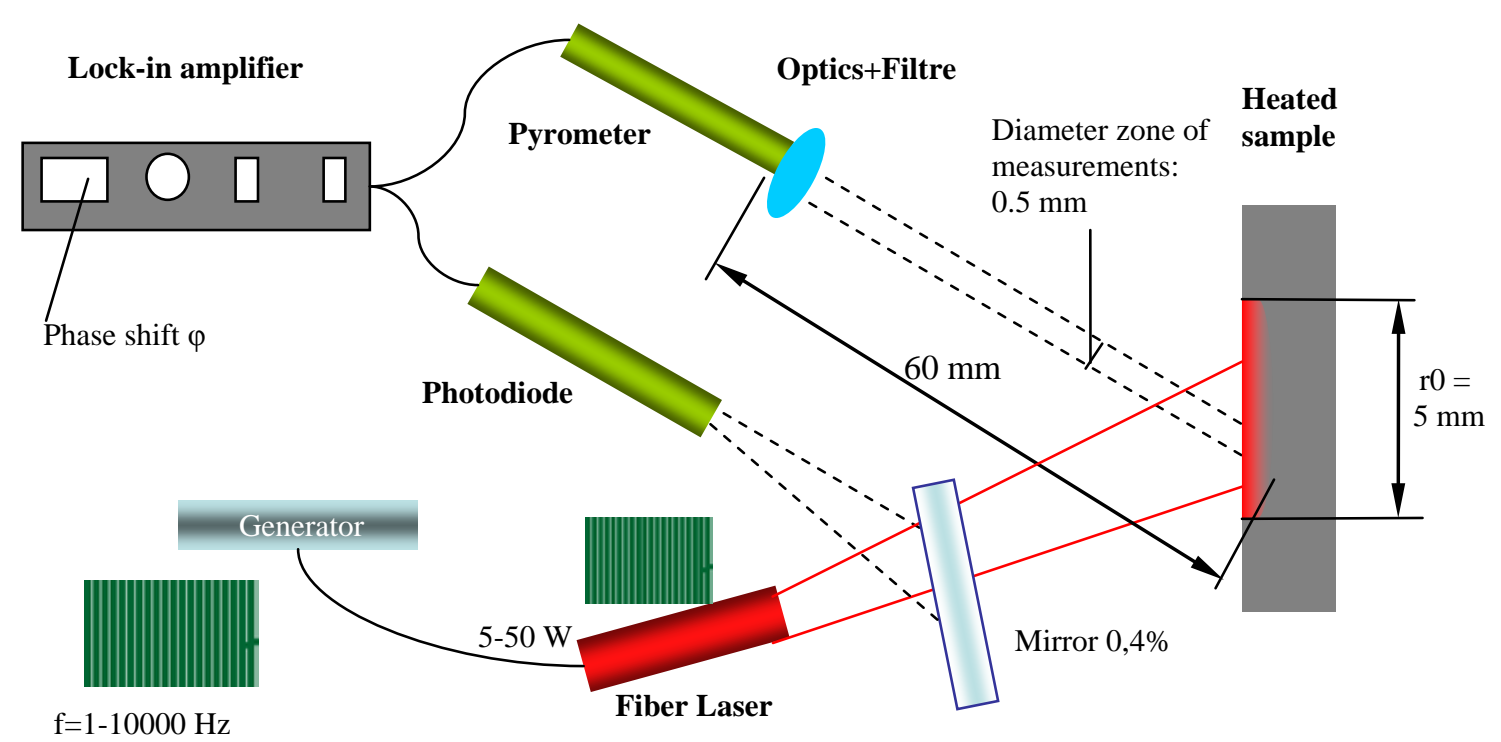

Figure 2. Schematic view of experimental setup. 


\section{Model validation}

In order to verify the theoretical model, two series of measurements were performed on thin sheets of stainless steel with thickness of 180 and $250 \mu \mathrm{m}$. We have measured the phase lag between photodiode and pyrometer signals. To obtain higher signal-to-noise ratio of the pyrometer, the samples is heated to temperatures $300-400{ }^{\circ} \mathrm{C}$. Then we have simulated a theoretical curve with best fitting of experimental points.

In this case the substrate is considered as air. The thermal properties of air are supposed known and constant (see Table 1). The laser beam radius at the level $1 / \mathrm{e}$ is $1.8-1.9 \mathrm{~mm}$. The thermal contact between sample and air is supposed to be perfect.

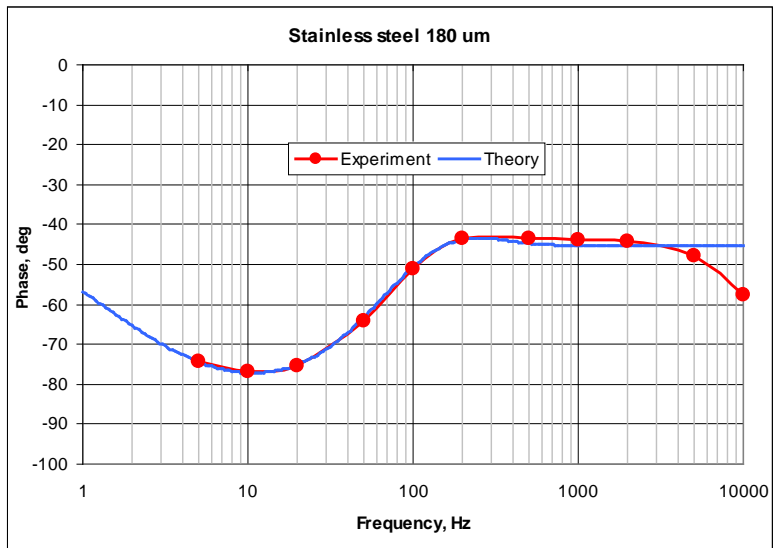

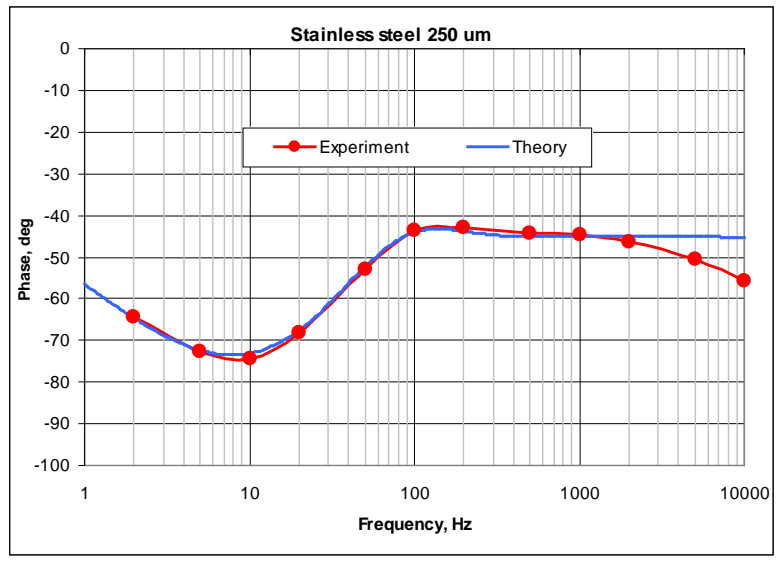

Figure 3. Phase shift on the stainless steel sheets with 180 (a) and 250 (b) $\mu \mathrm{m}$ thickness.

Table 1. Thermophysical properties of stainless steel sheets.

\begin{tabular}{|c|c|c|c|c|}
\hline Parameter & Symbol & \multicolumn{2}{|c|}{ Optimized values } & $\begin{array}{c}\text { Theoretical values } \\
@ 300^{\circ} \mathrm{C}\end{array}$ \\
\hline \multicolumn{2}{|l|}{ Layer } & SS 1 & SS 2 & Stainless steel \\
\hline Layer thermal conductivity, W/mK & $\mathrm{k}_{\mathrm{C}}$ & 26 & 24 & 20 \\
\hline Layer thermal capacity, $\mathrm{MJ} / \mathrm{m}^{3} \mathrm{~K}$ & $\mathrm{c}_{\mathrm{c}} \rho_{\mathrm{C}}$ & 3.6 & 3.6 & 4.3 \\
\hline Absorption coefficient, $1 / \mu \mathrm{m}$ & $\alpha$ & 20 & 20 & $20-50$ \\
\hline Layer thickness, $\mu \mathrm{m}$ & $\Delta$ & 250 & 180 & - \\
\hline \multicolumn{2}{|l|}{ Substrate (Air) } & \multicolumn{2}{|c|}{ Air } & Air @ $300^{\circ} \mathrm{C}$ \\
\hline Substrate thermal conductivity, W/mK & $\mathrm{k}_{\mathrm{s}}$ & \multicolumn{2}{|c|}{0.03} & $0.02-0.05$ \\
\hline Substrate thermal capacity, $\mathrm{MJ} / \mathrm{m}^{3} \mathrm{~K}$ & $\mathrm{Cs}_{\mathrm{S}} \rho_{\mathrm{s}}$ & \multicolumn{2}{|c|}{0.001} & 0.001 \\
\hline \multicolumn{5}{|l|}{ Others } \\
\hline Contact thermal resistance, $\mathrm{m}^{2} \mathrm{~K} / \mathrm{W}$ & $\mathrm{R}$ & \multicolumn{2}{|c|}{$10^{-9}$} & - \\
\hline Laser radius $1 / \mathrm{e}, \mathrm{mm}$ & $r_{0}$ & 1.9 & 1.8 & $1.4-2.1$ \\
\hline
\end{tabular}

The manually optimized theoretical curve is in good agreement with experimental results. But, more important, both samples can be described by similar thermophysical properties almost equal to the values of stainless steel found in literature. It means that our theoretical model predicts the thermophysical properties of a homogeneous sheet with good accuracy.

However it should to be mentioned that there is some discrepancies for high frequencies above $2 \mathrm{kHz}$. This could be explained by the volume emission caused by surface roughness.

\section{Results ITER-like and Discussion}

The main goal of our work is to study samples with a surface layer similar to that obtained in present and future fusion reactors of tokamak type. In some of them graphite is used as basic chamber wall material. In others it could be beryllium or tungsten. Because of the difficulty to work with beryllium for laboratory experiments it can be simulated by aluminum.

Tested Samples were made with a deposited $10 \mu \mathrm{m} \mathrm{Al} / \mathrm{C}$ layer by plasma enhanced chemical vapor deposition method on the polished graphite substrate $(30 \times 30 \times 5 \mathrm{~mm})$. 

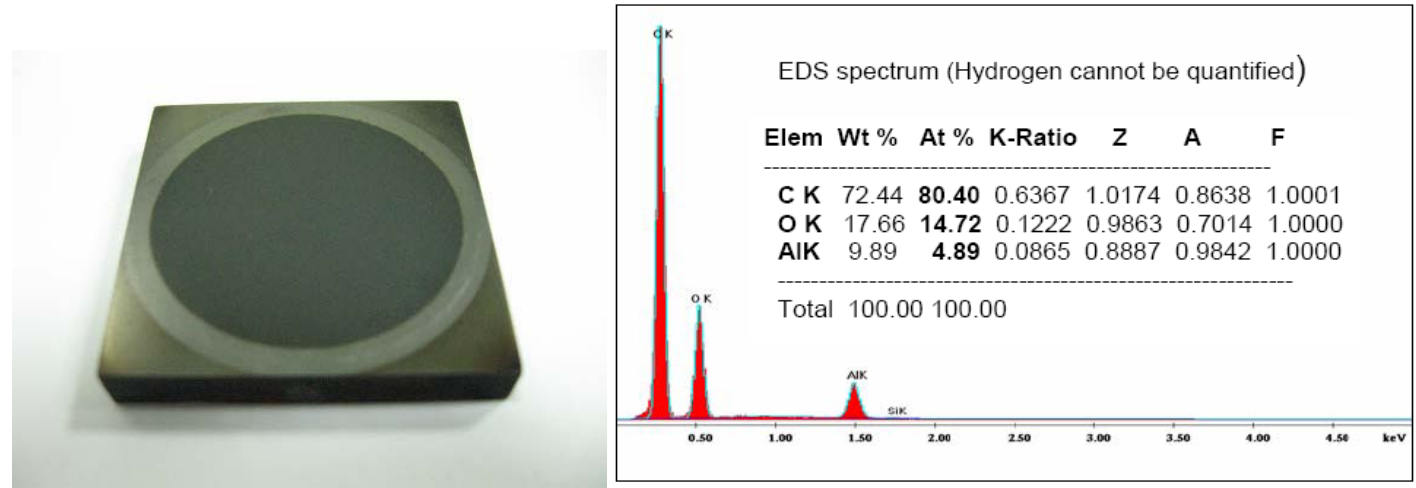

Figure 4. ITER-like sample view and their chemical composition.

First we measured the phase shift on the simple substrate without any deposited layer to determine the thermophysical properties of graphite.

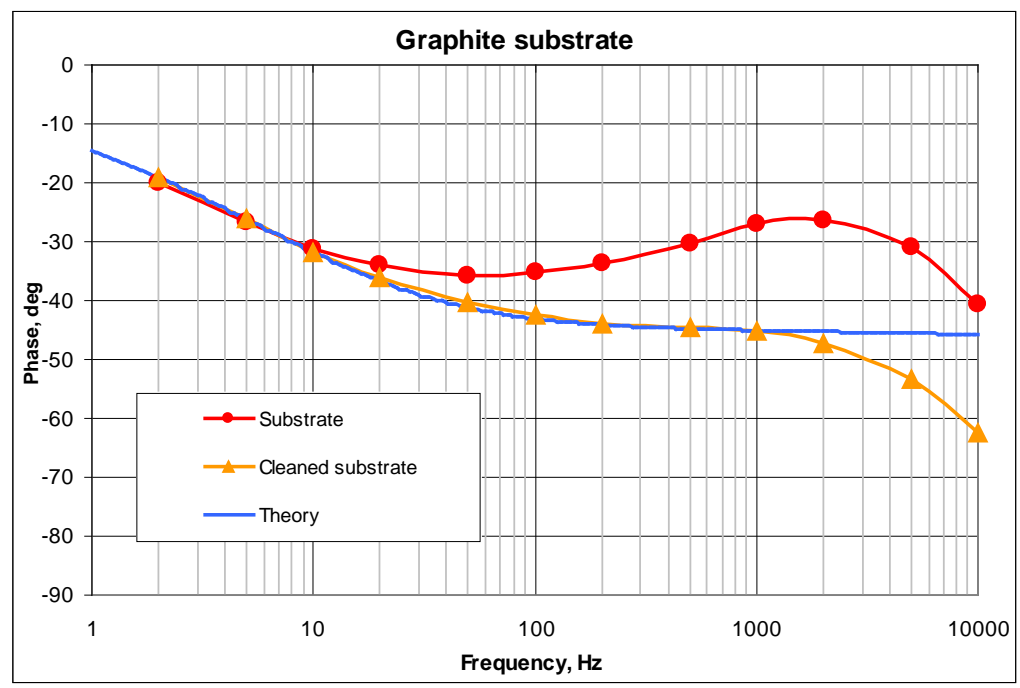

Figure 5. Phase shift measurements of graphite substrate.

Normally homogeneous semi-infinite substrate has a phase shift of -45 degrees before 3D thermal regime region (frequencies below $100 \mathrm{~Hz}$ for our system). It is not the case of our sample (see curve Substrate at figure 5). Therefore some additional layer is possibly present on the surface of our graphite substrate. To prove this we ablated the surface of the sample with a laser. Curve Cleaned substrate on the figure 5 represents measurements on the treated zone. Fitting of this experimental curve by the theoretical model gives us a value of diffusivity $a=4 * 10^{-5} \mathrm{~m}^{2} / \mathrm{s}$ (curve Theory on the figure 5). Together with a measured density of a sample $1670 \mathrm{~kg} / \mathrm{m}^{3}$ and theoretical thermal capacity of carbon $\mathrm{c}=1500 \mathrm{~J} / \mathrm{kg}^{\star} \mathrm{K}$ it is possible to determine the thermal conductivity: $\mathrm{k}=100 \mathrm{~W} / \mathrm{m}^{*} \mathrm{~K}$.

This superficial layer of substrate can be simulated in the sample with Al/C layer as not perfect thermal contact. But strictly speaking if the thermal contact at the form of contact thermal resistance $R\left[\mathrm{~m}^{2} \mathrm{~K} / \mathrm{W}\right] \mathrm{describes}$ adequately only intermediate layer with poor thermal capacity (e,g. air). If not it should be described and introduced into the model like third layer. Study of three and more layers system will be investigate in future experiments.

The result of measurements on the sample with deposited layer is presented on the Fig 6 . The optimized parameters of the layer and thermal contact between layer and substrate are in the table 2. Average time of calculations for one theoretical curve is less than 1 second.

Note that the layer has thermal capacity near to the theoretical one for carbon and aluminum, but thermal conductivity and light absorption coefficient are very poor. It can be explained by great oxidation of aluminum while deposition process.

There is still a little discrepancy in the region of high frequencies that could be explained by surface roughness. This phenomenon is not included in our model, but it is for further development. 


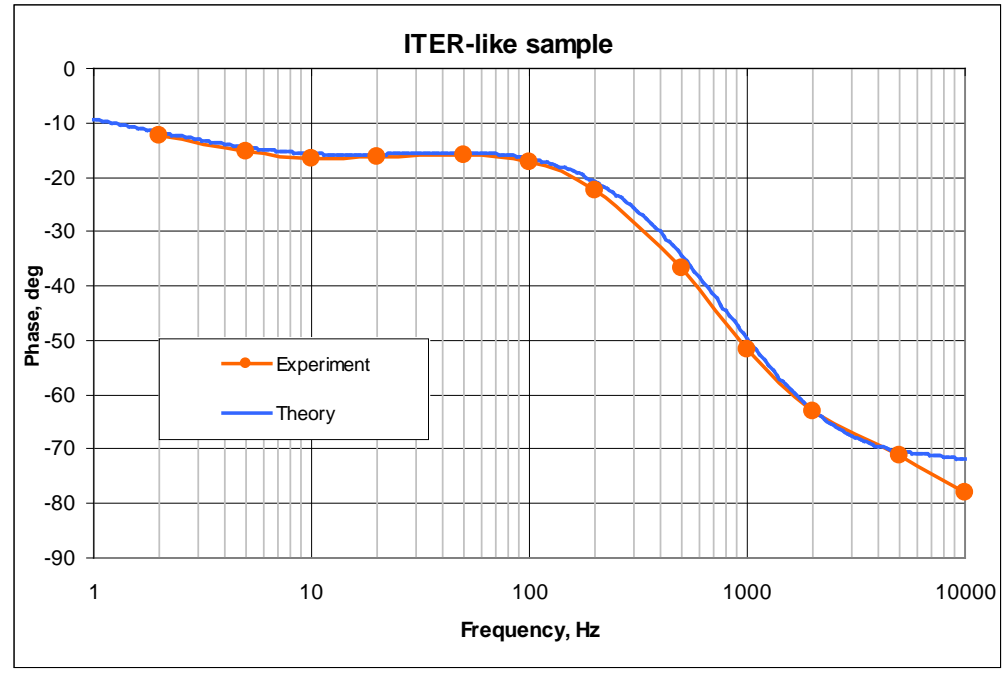

Figure 6. Phase shift of ITER-like sample.

Table 3. Thermophysical properties of ITER-like sample.

\begin{tabular}{|c|c|c|c|c|}
\hline Parameter & Symbol & Optimized values & \multicolumn{2}{|c|}{$\begin{array}{c}\text { Theoretical values } \\
@ 300^{\circ} \mathrm{C}\end{array}$} \\
\hline \multicolumn{3}{|c|}{ Layer } & C & Al \\
\hline Layer thermal conductivity, W/mK & $\overline{\mathrm{k}_{\mathrm{c}}}$ & 2 & $60-120$ & 237 \\
\hline Layer thermal capacity, $\mathrm{MJ} / \mathrm{m}^{3} \mathrm{~K}$ & $\mathrm{c}_{\mathrm{C}} \rho_{\mathrm{C}}$ & 2.5 & 3.37 & 2.43 \\
\hline Absorption coefficient, $1 / \mu \mathrm{m}$ & $\alpha$ & 0.2 & 2 & 20 \\
\hline Layer thickness, $\mu \mathrm{m}$ & 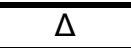 & 11 & \multicolumn{2}{|c|}{10} \\
\hline \multicolumn{3}{|c|}{ Substrate } & \multicolumn{2}{|c|}{$\mathbf{C}$} \\
\hline Substrate thermal conductivity, W/mK & $\overline{k_{s}}$ & 100 & \multicolumn{2}{|c|}{$60-120$} \\
\hline Substrate thermal capacity, $\mathrm{MJ} / \mathrm{m}^{3} \mathrm{~K}$ & $\mathrm{CS}_{\mathrm{S}} \rho_{\mathrm{S}}$ & 2.5 & \multicolumn{2}{|c|}{3.37} \\
\hline \multicolumn{3}{|c|}{ Others } & & \\
\hline Contact thermal resistance, $\mathrm{m}^{2} \mathrm{~K} / \mathrm{W}$ & $\bar{R}$ & $4.8 \times 10^{-6}$ & \multicolumn{2}{|c|}{ - } \\
\hline Laser radius $1 / \mathrm{e}, \mathrm{mm}$ & $\mathrm{r}_{0}$ & 1.8 & \multicolumn{2}{|c|}{$1.7-1.9$} \\
\hline
\end{tabular}

The determined parameters are to be used for estimation of laser ablation regimes or temperature of heating to make tritium vanishing from surface layer. So it is important to know layer and substrate parameters with precision in order not to overheat the surface but reach high enough temperatures.

The precision of phase shift measurements with lock-in amplifier is less than 1 degree for frequencies of 10-1000 Hz and about 1-2 degrees for low and high frequencies. This error has an influence on variation of optimized parameters about $5-10 \%$ and it is a sufficient precision. The verification of surface temperatures (i.e. amplitudes) with obtained parameters is follows.

\section{Conclusion}

The presented method of phase shift measurements is a suitable technique of contactless characterization of thin layer thermal properties. It dispense of knowing the emissivity of the surface for pyrometer measurements.

The developed theoretical model of fast phase shift calculation allows determining thermo-physical properties of deposited layer with enough precision and short time of calculations. The presented model is in good agreement especially with substrate-layer systems similar to introduced by hypothesis (one homogeneous layer on the semi-infinite substrate). For further development of the theoretical model it is necessary to include a surface roughness of the layer and third layer instead of intermediate thermal contact.

Concerning the experiments it is essential to compare the heating simulation with obtained parameters and real thermal evolution on the surface. In other words it is a study of amplitudes.

\section{REFERENCES}

[1] S. Fomichev, A. Semerok, J.-M. Weulersse and F. Brygo, Modelling of complex surface heating by continuous and pulsed lasers, report CEA, NT DPC/SCP 05-109-A, January 2005, 76 pages.

[2] A. Semerok, F. Jaubert, S. Fomichev, P.-Y. Thro, X. Courtois and C. Grisolia, Phase lock-in laser active pyrometry for surface layer charaterisation, report CEA, NT DPC/SCP 09-301-A, February 2009, 98 pages. 